Caries Res. 1972;6:379-380

\title{
Subject Index Vol. 6
}

Apatite, synthetic 1

Carbohydrate, dietary 52 Carbonate, dietary 183 Caries 156, 169, 229, 244

and diet 355

etiology 334

-, $\quad$ experimental $16,43,52,289$

-, $\quad$ monkeys 43

-, $\quad$ rats $52,183,203$

-, $\quad$ secondary 16

Demineralisation 334 Dental plaque 103 Dentifrice 237 Dentine, carious 60 Developmental disturbances 224 Dextranase 132, 289 Diet 244

- $\quad$ and caries 355

Enamel 156, 169, 334, 346

biopsy 193

hypoplasia 224

surface 312 Enolase inhibition 93 Enzyme kinetics 93 Epidemiology 229, 355 Experimental caries 203, 211, 244

Fluoride 1,93,237,244 -, bone 183 -, dietary 183

distribution 312

in enamel 193

mouth rinse 325

Glycerophosphate 43 Gnotobiosis 203, 211

Hamster 244 Histochemistry 60 Histopathology 16, 156, 169

Invertase 122 Irradiation 298

Lactate 334

Membrane potential 346

- $\quad$ transport 122

Microbiology 211

Microflora, oral 298

Mineral additives 244

Mineralisation 224

Monofluorophosphate 1

Mouth rinses 325

Oral fluoride absorption 325

Phosphate, dietary 183

380

Subject Index

Phosphate, microbial 34 Plaque 34, 289

analysis 103 
carbohydrates 103

matrix 203

polysaccharides 132 Polysaccharide hydrolysis 132 Polysaccharides in plaque 103

Saliva 148 Salivary flow 289 Streptococci 132

Streptococcus mutans 34, 122, 203, 211, 289, 298

- $\quad$ sanguis 211

Sucrases 122

Tribology 312 Tooth enamel 193

mineral 34

wear 312 Toxicity 237

Veillonella alcalescens 211 